\title{
Loss of CNOT9 begets impairment in gastrulation leading to embryonic
} lethality

Hemanta Sarmah1, Kentaro Ito², Mari Kaneko³, Takaya Abe², Tadashi Yamamoto ${ }^{*}$

${ }^{1}$ Cell Signal Unit, Okinawa Institute of Science and Technology, Kunigami, Okinawa 9040412, Japan

2 Division of Oncology, Department of Cancer Biology, Institute of Medical Science, University of Tokyo, Tokyo 108-8639, Japan

${ }^{3}$ Laboratory for Animal Resources and Genetic Engineering, RIKEN BDR, 2-2-3 Minatojimaminamimachi, Chuou-ku, Kobe 650-0047, Japan

* Correspondence: hemanta.sarmah@oist.jp 


\begin{abstract}
:
The multi-subunit eukaryotic CCR4-NOT complex imparts gene expression control primarily via messenger RNA (mRNA) decay. Here, we present the role of subunit CNOT9 in target mRNA decay during embryonic development. CNOT9 null mice appear normal by the onset of gastrulation (E7.0), however, exhibit growth and differentiation defects accompanied by extensive cell death by embryonic day 9.5 (E9.5). Sox-2 Cre conditional CNOT9 knockout mice show almost identical phenotype with brief delay in onset and progression, suggesting defects to be epiblast-dominant. Among various identified targets, we show that Lefty2 mRNA expression is post-transcriptionally regulated by CNOT9. Lefty2 3'-UTR containing mRNA has significantly higher stability in cells expressing mutant form of CNOT9, relative to cells expressing wild-type CNOT9. In addition, CNOT9 primarily localizes within the cytoplasm and bridges interactions between the CCR4-NOT complex and miRNA-RISC complex in gastrulating embryos.
\end{abstract}




\section{Introduction:}

Messenger RNA (mRNA) decay is an indisputable component of gene expression regulation. In higher eukaryotes such as humans and mice, a conserved multi-subunit protein complex, known as CCR4-NOT, interacts with RNA binding proteins and collectively performs mRNA decay (Chapat and Corbo, 2014, Shirai et al., 2014, Collart, 2016, Miller and Reese, 2012). The complex is composed of a central scaffold CNOT1, regulatory subunits CNOT2 and CNOT3, a catalytic core consisting of CNOT6 or CNOT6L and CNOT7 or CNOT8 proteins, and three additional subunits CNOT9, CNOT10, and CNOT11 (Shirai et al., 2014). Precise molecular function of subunits CNOT9-11 is yet to be understood. In this study, we elucidate the molecular and physiological function of murine CNOT9 with regard to mechanisms on mRNA decay during embryonic development.

Embryonic development is a complex, yet fine-tuned orchestration of gene expression regulation (Reik, 2007, Tomancak et al., 2007, Wang et al., 2005, Yi et al., 2010). Germ layer differentiation, also known as gastrulation, is an event that takes place during embryonic day 6.5 to 8.5 of post-implantation mouse embryos (Gilbert, 2010). Cell lineage fates are determined during this stage based on various extracellular signaling molecules and chemical gradients (Basson, 2012, Hammerschmidt and Wedlich, 2008, Wang et al., 2012). With the help of targeted gene disruption methods in mice, various genes have been identified as key determinants indispensable for embryonic gastrulation. While a majority of studies have shown transcription regulation as the underlying mechanism by which these genes influence gastrulation, the post-transcriptional component has not been adequately investigated. In this study, we present a scenario of post-transcriptional regulation of a gastrulation related gene Lefty2, whose expression is controlled by CCR4-NOT complex subunit CNOT9.

In-vitro, CNOT9 (also known as Rqcd1) was first shown to mediate retinoic-acid (RA) induced differentiation of F9 teratocarcinoma cells by positively influencing $c$-jun transcription (Hiroi et al., 2002). In the same study, increased expression of CNOT9, as a result of RA treatment, was found to rather inhibit branching morphogenesis of embryonic lung, via unknown mechanisms (Hiroi et al., 2002). In other studies, CNOT9 was shown to have physical interactions with non CCR4-NOT components - c-Myb and NIF-1 proteins, resulting in transcription inhibition and ligand-dependent activation of RARs and RXRs, respectively (Garapaty et al., 2008, Haas et al., 2004). Recently, CNOT9 has been confirmed to be a core 
component of the CCR4-NOT complex through crystal structure analysis and has been implicated in microRNA mediated RNA decay (Mathys et al., 2014, Chen et al., 2014). Therefore, with such heterogeneity in knowledgebase around CNOT9, we chose to investigate its physiological relevance and molecular function. We characterized the phenotype exhibited by CNOT9 knock-out (KO) embryos, and its role in specific mRNA decay during gastrulation.

\section{Results:}

\section{Loss of CNOT9 causes defects in embryonic gastrulation:}

We generated Cnot9+/Lacz mice as described in Materials and Methods (Fig.S1b). Although Cnot9+/Lacz mice appeared phenotypically normal, upon crossing of male and female Cnot9 $^{+/ L a c Z}$ mice no Cnot9LacZ/LacZ (KO) mice were born. Furthermore, no KO embryos could be genotyped at embryonic day 14.5 (E14.5), suggesting early embryonic lethality. To identify the timing of embryonic lethality and onset of phenotypic defects in $\mathrm{KO}$ embryos, we investigated gastrulation stage embryos, 7 to 10 days post coitum. During early Bud/Headfold stages (E7.25 to E7.75) and early somite stages (E7.75 to E8.0), no obvious differences in KO embryo morphology were observed when compared with wildtype (WT) and heterozygous (HE) littermates (Fig.1a,1b). However, during intermediate and late somite stages (E8.0 to E8.5), CNOT9 KO embryos displayed onset of phenotypic defects in the form of growth retardation of embryo-proper (Fig.1c). On the following embryonic day, these KO embryos showed severe growth defects characterized by small size, perturbed developmental pace indicated by delay in embryo curling, and lack of blood vessels within visceral yolk-sac regions (Fig. 1d). Contrary to the previous stage, KO embryos exhibited higher cleaved PARP expression compared to WT littermates, suggesting cell death within these embryos (Fig. 1d). Another day into development, $\mathrm{KO}$ embryos continued to display severe defects in terms of embryo size and morphology and showed a sustained increase in cleaved PARP expression compared to WT littermate controls (Fig. 1e). Mouse embryonic stem cells derived from KO blastocysts were viable and did not show any defects in terms of colony size, growth rates or expression of stemness markers, suggesting a gastrulation specific defect within $\mathrm{KO}$ embryos (data not shown).

\section{CNOT9 KO embryo phenotype primarily contributed by epiblast lineage cells:}

Due to the high prevalence of placental defects in embryonic lethal mutant mice, we decided to test the contribution of trophoblast vs. epiblast lineage cells in driving $\mathrm{KO}$ embryo phenotype (Perez-Garcia et al., 2018). We performed histological analysis on placental regions during 
E8.5 and E9.5 stages and found that KO embryos possessed significantly smaller sized placentas compared to WT and HE littermate controls (Fig. S2a, S2b). A reduced or poorly developed placenta may, however, be caused due to an overall impairment in embryo development and differentiation. To test this observation from the standpoint of direct vs. indirect outcome of CNOT9 loss, we generated Cnotgfloxfllox mice, as described in Materials and Methods (Fig.S1a). Mice containing floxed Cnot9 alleles were crossed with Sox2-Cre mice (B6.Cg-Edil3Tg(Sox2-cre)1Amc/J, Jackson Labs, USA) to generate epiblast specific knockout (eKO) mice. Similar to complete knockouts, no eKO mice were born suggesting embryonic lethality. Analysis of gastrulating eKO embryos during intermediate and late somite stages identified no characteristic morphological differences between WT controls (Fig. 1C). However, during late gastrulation stages, defects in eKO embryos began to surface, initially in regions of yolk-sac vasculature, followed by growth arrest and cell death in embryo-proper (Fig. 1c,1d).

\section{Cnot9 expression during embryonic gastrulation via LacZ staining:}

Taking advantage of the LacZ knock-in allele in CNOT9 HE embryos, we performed bgalactosidase (b-gal) staining of embryos between E7.5 to E9.5 stage of gastrulation (Fig.2). At E7.5 stage, b-gal staining was predominantly seen within the epiblast region that would eventually contribute to form the embryo-proper (Fig.2a). At 8.5 stage, b-gal staining was seen in various parts of the embryo-proper that include the neuroepithelium, the trunk region, and the caudal region of the embryo. More importantly, b-gal staining was also seen in the extraembryonic yolk-sac and within the ectoplacental cone (EPC) region of the embryo (Fig.2b). A day later, E9.5 stage embryos showed strong b-gal staining within labyrinthine and spongiotrophoblast regions of the placenta relative to weak and punctuated signal from regions of the embryo-proper (Fig.2c). A schematic representation of placental layers during E9.5 stage is depicted for visualization of lacZ expression within the placenta (Fig.2d). In addition to lacZ staining Cnot9 mRNA expression (relative to Gapdh) at various regions of gastrulating embryos, was determined by qRT-PCR analysis (Fig.2e). In agreement with the b-gal staining pattern, CNOT9 expression was about > 1.5 higher in E9.5 placenta compared to embryo-proper. Other stages showed nearly similar expression levels of CNOT9 mRNA.

\section{Gene expression regulation via CNOT9:}

To investigate the molecular function of CNOT9 with added impetus from the KO embryo phenotype, we performed RNA-Seq analysis on three independent E8.0-E8.5 staged WT and KO samples. From a total of 15534 protein-coding targets, only 384 were upregulated $>2$-fold, while another 338 were downregulated $>2$-fold in KO embryos based on normalized RPKM 
scores (Fig.3a). Top few upregulated targets included Lefty2, Lefty1, Nodal, Crypto-1, and Noto that have previously been known for their role in embryonic gastrulation. A heatmap of a few select upregulated targets and downregulated targets are shown in Fig. 3b and Fig. S3a respectively. These in-silico results were further validated by qRT-PCR based analysis using E8.5 stage WT and KO cDNA samples (Fig. 3c). With the exception of Noto, other validated targets that showed upregulation at E8.5 stage, also showed either a trend or significant increase during E7.5 stage. This suggests that despite the lack of phenotypic defects during E7.5 stage, molecular events leading to abnormal embryo phenotype had already perpetuated within $\mathrm{KO}$ embryos. Primers used for the same have been listed in Fig. S3c. Pathway analysis performed using Gene-Set Enrichment Analysis (GSEA) algorithm clearly identified Nodal and TGF-beta signaling pathways to be affected in $\mathrm{KO}$ embryos, thereby summarizing the nature of gastrulation defects (Fig. 3d). Downregulated targets mainly suggested retarded embryo growth and differentiation characterized by significantly reduced expression levels of $M y l 2$, Epo, Fgf8, Sox10 and Pax family members - Pax1, Pax5 and Pax7 (Fig. S3b). Downregulated targets didn't clearly suggest any specific signaling pathway (data not shown). In addition to upregulated targets involved in gastrulation, E8.5 stage KO embryos also showed higher expression for Oct4 and c-myb mRNAs (Fig. 3c). We speculate that such an increase is contributed by a higher abundance of cells in an undifferentiated state within $\mathrm{KO}$ embryos, compared to controls.

\section{CNOT9 contributes to Lefty1/2 mRNA decay, in-vitro:}

In agreement with the current understanding of CNOT9 being associated with the mRNA decay complex, we found the protein to be predominantly localized within cytoplasmic fractions of gastrulating embryos (Fig. S4a). Furthermore, CNOT9 participated in complex formation with CCR4-NOT complex subunits - CNOT1, CNOT2, and CNOT3, as well as RISC component subunit GW182 during gastrulation as observed in a co-immunoprecipitation assay performed with anti-CNOT3 antibody. (Fig. S4b). To investigate the role of CNOT9 in the context of mRNA decay, we pursued two candidate targets - Lefty1 and Lefty2, as validated in the previous section. Another important reason for picking these targets among others is the presence of miRNA binding sites within 3'UTR regions that is likely to facilitate mRNA decay, in-cis. Furthermore, we generated CNOT9 KO HeLa cells using CRISPR-Cas9 method for performing in-vitro biochemical assays. KO HeLa cells transfected with flaghCNOT9 or flag-hCNOT9mut4 were used to test the decay kinetics of reporter luciferase conjugated with Lefty2 or Lefty1 3'UTRs. ActinomycinD chase assay showed stabilization of both Lefty2 or Lefty1 3'UTR conjugated reporter mRNAs in KO cells reconstituted with 
hCNOT9mut4 over a period of 6 hours (Fig 4a). Western blotting performed using anti-CNOT9 and anti-flag antibodies confirmed equal dose of flag tagged CNOT9 protein expression (Fig 4c). c-myb 3'UTR known to possess multiple microRNA binding sites, clearly showed impaired reporter mRNA decay in the absence of CNOT9 (Fig 4a). p21(cdkn1a) 3'UTR conjugated reporter, used as a negative control, did not show any significant difference in decay kinetics (Fig 4b). Similar decay kinetics for hFHL3 mRNA (endogenous control) showed uniformity of ActinomycinD treatment across different samples, over the course of the experiment.

\section{Discussion}

The mammalian CCR4-NOT complex is profoundly involved in embryonic development. For instance, loss of CNOT3 leads to pre-implantation growth arrest in mouse embryos (Neely et al., 2010, Morita et al., 2011). In this study we highlight the physiological role of CNOT9 during embryonic gastrulation. Cnot9 knockout mice exhibited developmental abnormalities during mid-gastrulation stages (E8.0 to E8.5) leading to embryonic lethality at E9.5 stage. KO embryos showed a spectrum of defects that included reduced size, arrested growth, pale colorization of embryo-proper, impaired yolk-sac vasculature, and significantly reduced placenta. Chorioallantoic fusion defects were not observed in E8.5 KO embryos (data not shown). To determine the weightage contribution of epiblast vs. trophoblast derived cells, we examined the phenotype of embryos that underwent epiblast specific depletion of CNOT9. A marginal delay in the onset of phenotype and timing of embryo lethality was observed in Sox2Cre conditional knockout mice compared to complete knockouts. This suggested that phenotypic defects in $\mathrm{KO}$ embryos were mainly contributed by cells originating from the epiblast lineage. In other words, a wildtype trophoblast lineage could not profoundly rescue the phenotype observed in whole-body knockouts. As a first step towards examining function, we investigated the expression pattern of Cnot9 mRNA using whole-mount LacZ staining and qRT-PCR based analysis. Initially (E7.5), Cnot9 was almost exclusively expressed within the epiblast, but late in gastrulation, (E8.5 to E9.5) expression was also seen within placental regions. It is worth noting that both E8.5 and E9.5 placental regions showed higher expression of Cnot9 compared to relative levels within the adjoining embryo proper. Unfortunately, due to the timing and nature of defects, both $\mathrm{KO}$ and conditional knockout mice models limit our understanding of CNOT9 within a functional placenta. It would be worth investigating CNOT9 function within placental lineage via in-vitro trophoblast stem cell-based differentiation assays, or in-vivo models that produce placenta specific depletion. 
Phenotypic defects in KO embryos motivated us to address the molecular function of CNOT9 in developmental context. E8-E8.5 staged WT and KO embryos were pooled and subjected to RNA-Seq analysis. With biochemical validation, we observed significant upregulation in mRNA levels of Lefty1/2, Nodal, Cfc-1, among others that have previously been shown to have profound roles in embryonic development (Meno et al., 1998, Meno et al., 1999, Conlon et al., 1994, Yan et al., 1999). For instance, overexpression of Lefty2 in zebrafish embryos blocks head and trunk mesoderm formation (Meno et al., 1999). Cfc-1 overexpression in mice caused downregulation of genes involved in cardiac differentiation during mid-gastrulation stages whereas, in chick embryos, its gain-of-function led to suppression of posterior mesodermal fates while promoting anterior mesoderm development (Chu et al., 2005, Lin et al., 2016). Nodal overexpression has been shown to promote differentiation of mouse ES cells into endoderm and mesoderm lineages at the expense of neuroectoderm formation (Pfendler et al., 2005). Despite these findings, little is known from the standpoint of post-transcriptional regulation of such transcripts and their consequences on embryo development. One such report showed Lefty2 mRNA regulation via microRNA-127 in mouse ES cells (Ma et al., 2016). Therefore, we present a model where the physiological relevance of the upregulation of gastrulation related transcripts can be studied.

We focused on Lefty1 and Lefty2 transcripts mainly because of the extent of upregulation and the presence of canonical microRNA binding sites in their 3'UTR elements. With the help of TargetScan database, we found that both mRNAs contain an AAGCACU element that serves as binding sites for miR-291-3p, miR-294-3p, miR-295-3p, miR-302-3p microRNA. Therefore, we cloned 3'UTR elements in pGL3.0 luciferase expression vectors. In WT HeLa cells, relative to vector-only control, luciferase reporter expression and activity were significantly reduced when conjugated with Lefty1 and Lefty2 3'UTRs (data not shown). We further clarified that in absence of wild-type human CNOT9, mRNA decay of reporters containing Lefty1 or Lefty2 3'UTRs was significantly affected. Due to very high sequence conservation of human vs. mouse Cnot9 proteins (99.7\%), and microRNA binding sites within UTR elements of mouse and human Lefty transcripts, a similar mechanism is likely to be present in humans.

The pale color of E8.5 - E9.5 stage KO embryos can be explained in terms of dysfunctional waves of primitive and definitive waves of erythropoiesis depending on $c$-myb expression. Downregulation of $c$-myb transcription has previously been reported to be imperative for erythropoietin (epo) induced differentiation (Todokoro et al., 1988). Furthermore, retinoic acid has been shown to suppress c-myb expression (Mandelbaum et al., 2018). CNOT9 has 
previously been proposed to be retinoic acid-inducible and therefore, in agreement with these ideas, our data suggests a strong correlation in terms of loss of CNOT9, the elevation of $c$ myb expression, and reduced Epo expression (Hiroi et al., 2002). The difference, however, lies in the manner in which c-myb mRNA expression is elevated. These two evidences showed transcriptional control in $c-m y b$ expression, while our data suggests post-transcriptional control (via mRNA stabilization) in the backdrop of CNOT9 loss. Although defects in blood formation may be triggered by improper germ layer differentiation at earlier stages of gastrulation, we speculate that elevation of $c-m y b$ mRNA can inhibit epo induced erythroid differentiation in CNOT9 KO or conditional knockout mice. Further analysis using mice models for erythroid-specific deletion can help elucidate this aspect of CNOT9 function.

CNOT9 has been proposed to play regulatory roles within the complex in a manner that assists mRNA decay by interacting with CNOT1 and stimulating catalysis by subunits CNOT6, CNOT6L, CNOT7 and CNOT8 (Pavanello et al., 2018). This idea was further perpetuated by an in-vitro study of the human CCR4-NOT complex, which suggested that stimulus-dependent CNOT9 interaction with RNA binding proteins results in targeted mRNA decay (Raisch et al., 2019). In congruity with this idea we observed a similar trend within our RNA-Seq data. Only 722 (less than $5 \%$ ) out of 15534 total detected protein-coding genes underwent more than 2fold significantly change. In other words, developmental stimulus affects the expression of very specific targets via CNOT9, leaving behind a majority of them unaffected.

The molecular function of CNOT9 in regulating target mRNA decay either by microRNA pathway or via interactions with RNA binding proteins, demands further investigation. Recently, two studies had shown that interactions between GW182 proteins with CNOT1-CNOT9 complex facilitate miRNA mediated decay (Chen et al., 2014, Mathys et al., 2014). Their findings suggested that the presence of CNOT9 provided structural stability to the trimeric complex and augmented the affinity of GW182 for CNOT1. Because their experiments were performed either in cell-free conditions or in wild-type HEK-293T cells that may express endogenous levels of CNOT9, we tested this theory using CNOT9 KO HeLa cells. We found that absence of CNOT9 completely abrogated interactions between the C-terminal silencing domain of GW182 with CCR4-NOT subunits CNOT1 and CNOT3 (Fig. S5). This makes CNOT9 an indispensable element within the molecular framework of microRNA mediated mRNA decay. 


\section{Materials and Methods:}

\section{Generation of Cnot9-knockout and Cnot9 conditional mice:}

Targeting vector used for generation of Cnot9-knockout mice (Accession. No. CDB0573K: http://www2.clst.riken.jp/arg/mutant\%20mice\%20list.html) was constructed from a 129SVJ genomic clone (STRATAGENE, CA). Exon 1 of the Cnot9 locus was targeted and replaced by LacZ and neomycin resistance gene by electroporating TT2 ES cells (Yagi et al., 1993). Neomycin-resistant ES cell clones harboring the homologous recombination were screened by PCR and Southern blot analysis. Two correctly targeted ES cell lines were injected into ICR 8-cell-stage embryos to generate chimeric mice. Germline-transmitted male chimeras were crossed with C57BL/6J female mice (Japan CLEA, Tokyo) to obtain heterozygous F1 offspring. Genotyping was performed using: 5'-TTATCTGGACGCGGGTTGTGAATGCTGG3' (Pr1), 5'-ACTAGTTCTAGAGCGGCCGATTTAAATACG-3' (Pr2) and 5'GTCCTAAGAAAGACATTCCAGGTAGAG-3' (Pr3) (Fig.S1c). Cnot9 conditional KO (Cnot9 $\left.{ }^{f / / f l}\right)$ mice (Accession. No. CDB1112K) were also generated similarly by targeting TT2 ES cell lines. To generate conditional alleles (floxed alleles) from targeted alleles, mice with targeted alleles were crossed with mice expressing FLP (Jackson \#009086). Genotyping was performed using: 5'- CATGGGCTCATTAGCTGTCAAACAGGTTGAG-3' (Pr1), and 5'CCACTGATAGATCTTCTCTCTGTCCACTTGG-3' (Pr2) (Fig.S1d). Experiments were performed with mice that had been backcrossed successively to C57BL/6J mice for at least seven generations. Animals were maintained in a 12-hour light dark-cycle within a temperature-controlled $\left(22^{\circ} \mathrm{C}\right)$ barrier facility with abundant food (Rodent Diet CA-1, CLEA Japan) and water. All animal experiments were carried out following guidelines for animal use issued by Animal Resources Section, OIST Graduate University and the Institutional Animal Care and Use Committee (IACUC), RIKEN Kobe Branch.

\section{Antibodies:}

Antibodies against CNOT1 and CNOT3 have been previously described (Chen et al., 2011). Commercially available antibodies used in this study were: anti-a-tubulin (Sigma, T9026), antiPARP (CST, 9542S), anti-CNOT9 (Proteintech, 22503-1-AP), anti-Flag (MBL, PM020), antiXrn-1(Bethyl A300-443A), anti-HistoneH3 (CST, 4499S), anti-GW182 (Bethyl, A302-329A), anti-CNOT2 (CST, 34214S), anti-4EBP1 (CST, 9644S), anti-b-actin (CST, 4970L), antiGAPDH (CST, 2118L), anti-CNOT10 (Bethyl A304-899A), and anti-Ago2 (CST, 2897S). 


\section{Tissue staining and histology:}

Post dissection, placental tissue was fixed in 4\% paraformaldehyde (PFA) solution overnight, followed by stepwise dehydration in Ethanol solutions and finally embedded in paraffin. 8$10 \mu \mathrm{m}$ sections generated using Microm HM325 rotary microtome were stained with Hematoxylin 3G (8656) and Eosin solutions (8659) (Sakura Finetek, Japan). For LacZ staining, E7.5, E8.5, and E9.5 stage embryos were first fixed in ice-cold $0.2 \%$ Glutaraldehyde solution of a period of 5,10 and 15 minutes respectively, followed by X-gal staining at $37^{\circ} \mathrm{C}$ for 48 hours. Whole-mount LacZ stained embryos were re-fixed in 4\% PFA solution overnight, embedded in JB4 resin (Polysciences, Inc.) and sectioned (10um) using Microtome Rotatif, HM335E.

\section{Mouse embryo and tissue section imaging:}

All images for mouse embryos across various stages were taken using Leica IC80 HD stereomicroscope. Tissue sections of embryo and placenta were imaged using Keyence BZX710 microscope.

\section{Plasmids:}

Flag-tagged human CNOT9 was cloned into pCDNA3.0 and CNOT9mut4 (H58A F60A A64Y V71Y) was generated using point mutagenesis. Mouse Lefty1 3'-UTR (425 bp), Lefty2 3'-UTR (1290 bp), c-myb 3'-UTR (1243 bp), were cloned into the pGL3 control vector. p21 3'-UTR in pGL3.0 control vector was generated by Dr. Akinori Takahashi in the laboratory. Flag-tagged full-length silencing domain of human GW182/TNRC6C (1260-1690 amino acids) was PCR amplified from RIKEN cDNA library (KIAA1582) and cloned into pCDNA3.0 vector.

\section{Generation of CNOT9 KO Hela cells with CRISPR/Cas9:}

Guide RNA sequences were designed using CHOP-CHOP website, provided by the University of Bergen, Norway (Labun et al., 2019). Insert oligonucleotides targeting exon1 of hCNOT9 were 5'-CACCGGCACAGCCTGGCGACGGCTG-3' and 5'AAACCAGCCGTCGCCAGGCTGTGCC-3'. These complementary oligos were annealed and cloned into pSpCas9(BB)-2A-Puro vector (Addgene). Plasmids containing guideRNA target sites were confirmed by sequencing. HeLa cells were transfected using pSpCas9(BB)-2A-Puro vectors containing guide RNAs that target hCNOT9 (test) or EGFP (control), as described previously (Ran et al., 2013). 24 hours post transfection, cells were treated with $2.5 \mu \mathrm{g} / \mathrm{ml}$ of puromycin for three days. After 3 days, cells were collected, and seeded in three $10 \mathrm{~cm}$ plates at a density of 300 cells per dish. After 10-12 days of culture, 
small colonies were picked and seeded into individual wells of a 48 well plate and propagated further. Knockout clones were confirmed by DNA sequencing and Western blotting.

\section{Cell Culture:}

HeLa cells were cultured in DMEM (low Glucose) containing 10\% FBS. TransIT-LT1 (Mirus Bio) reagent was used for transient transfection assays based on manufacturer's protocol. For determination of reporter mRNA half-life kinetics CNOT9 KO HeLa cells were first transfected with flag-hCNOT9 or flag-hCNOT9mut , for 24 hours, followed by transfection of luciferaseLefty2 3'-UTR mRNA, luciferase-Lefty1 3'-UTR mRNA, or luciferase-c-myb 3'-UTR mRNA for 4 hours. Cells were then treated with $2.5 \mathrm{ug} / \mathrm{ml}$ ActinomycinD (Wako) for 6 hours with samples being collected at 0,3 and 6 hours after treatment.

\section{Quantitative RT-PCR:}

Total RNA was isolated from mouse embryos and HeLa cells using Isogen II reagent (Nippongene), followed by cDNA synthesis using with SuperScript Reverse Transcriptase III Kit (Invitrogen), based on manufacturers protocol. For real-time PCR, cDNA (1:10 diluted) was mixed with target-specific primers and SYBR Green Supermix (Takara). Data analysis was done using Viia7 sequence detection interface (Applied Biosystems). mRNA expression for various targets was determined relative to mouse or human GAPDH levels using $\triangle \triangle \mathrm{Ct}$ method.

\section{Western Blotting:}

Western Blotting for various proteins/targets was done via detection of chemiluminescence (Amersham Bioscience) as described previously (Takahashi et al., 2015). Embryos or cultured cells were solubilized in TNE buffer (50 mM Tris-HCl [pH 7.5], $150 \mathrm{mM} \mathrm{NaCl}, 1 \mathrm{mM}$ EDTA, $1 \%$ NP40, and $1 \mathrm{mM} \mathrm{PMSF}$ ) for 10 minutes at $4^{\circ} \mathrm{C}$, after performing 10-second pulse homogenization of pellet (Fisherbrand Pellet Pestle). Total protein concentration was determined using BCA assay (Thermo Fisher), and adjusted by addition of appropriate amount of SDS sample buffer to lysates. Lysates were subjected to SDS-PAGE electrophoresis and electro-transferred into PVDF (polyvinylidene difluoride) membranes. Bands corresponding to various proteins were detected by corresponding antibodies using ImageQuant LAS 4000 (GE Healthcare, Tokyo) and analyzed using ImageQuant software. Nuclear and cytoplasmic fractions were isolated using the NE-PER kit (Thermo Scientific 78833) according to the manufacturer's protocol. 


\section{Protein Immunoprecipitation:}

E8.5 embryo lysates were subjected to immunoprecipitation using anti-CNOT3 antibody or mouse control IgG (Santacruz, sc-2025) for 1 hour at $4^{\circ} \mathrm{C}$, followed by an additional 1-hour incubation with $50 \mu \mathrm{l}$ of Dynabeads (Invitrogen) added to the antibody-lysate solution. Immunoprecipitated proteins were recovered by magnetic separation of bead-antibody-protein complex. Subsequently, individual proteins in immune complexes were immunoblotted with appropriate antibodies. HeLa cell lysates obtained after transfecting cells with flag-hCNOT9 or flag-hCNOT9mut4 or pCDNA3.0 vectors were subjected to immunoprecipitation using ANTIFLAG M2 Affinity gel (Sigma) for 2 hours at $4^{\circ} \mathrm{C}$. All lysates were subjected to BCA assay prior to IP, to ensure equal amount of total protein.

\section{RNA-Seq analysis:}

High-quality total RNA (500ng) was isolated from pooled E8.0-E8.5 stage embryos using Isogen II reagent (Nippongene) and subjected to RNA-seq library preparation using TruSeq Stranded mRNA LT Sample Prep Kit (Illumina) based on manufacturer's protocol. Paired-end RNA sequencing (109 base-pair) with a Hiseq PE Rapid Cluster Kit v2-HS and Hiseq Rapid SBS Kit v2-HS (200 Cycle) on a Hiseq2500 (Illumina) was performed following manufacturer's protocol. Sequencing reads were mapped to mouse reference genome provided in Ensembl database using StrandNGS software (Strand Life Sciences). Data analysis was done by mapping reads to the mm10 genome sequence (Ensembl), thereby converting raw counts to corresponding FPKM scores. For downstream analysis, only protein-coding genes with FPKM scores $\geq 0.05$ were selected. Gene set enrichment analysis (GSEA) was performed using online software from Broad Institute, USA. Sequence data are available through ArrayExpress under the accession number $\left(^{\star * \star *}\right)$.

\section{Statistical analysis:}

Unpaired, two-tailed Student's t-test was used for data analysis. Bar graphs represent mean \pm standard error of the means (SEM) and $p$-value $<0.05$ was deemed statistically significant. 


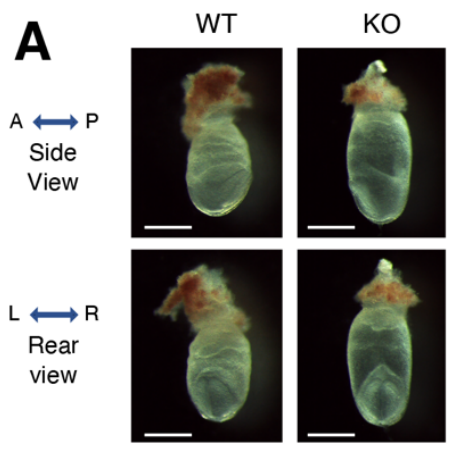

Bud/Headfold stage (E7.25- E7.75)

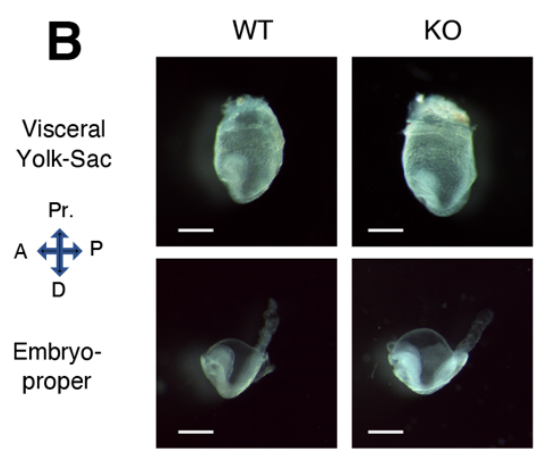

Early somite stages (E7.75 - E8.0)
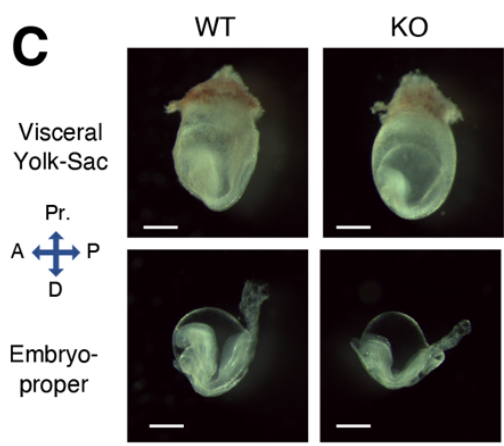

Sox2-Cre cKO
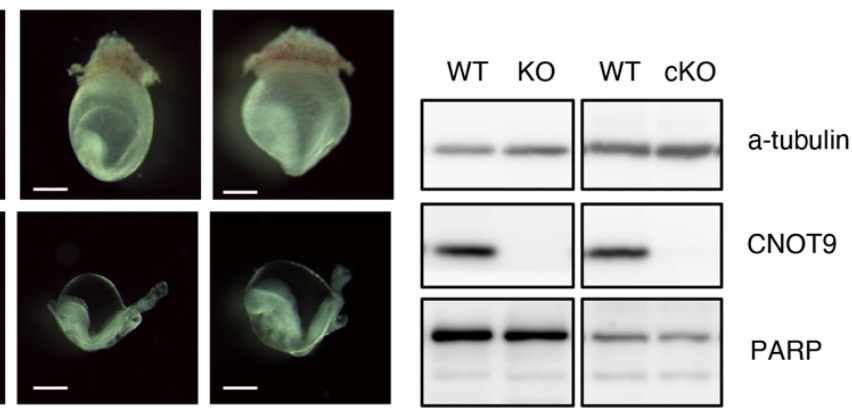

CNOT9

Intermediate to Late somite stages (E8.0 - E8.5)
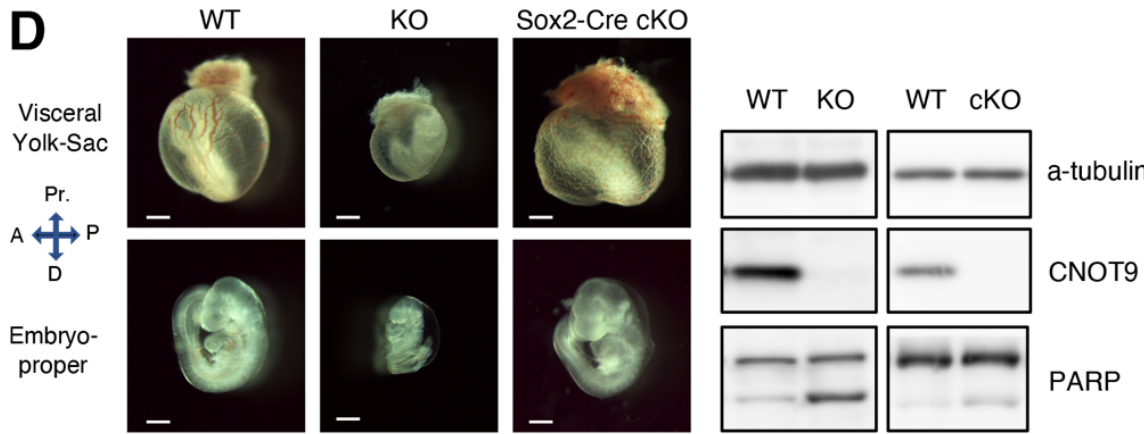

Late Gastrulation (E9.0- E9.5)
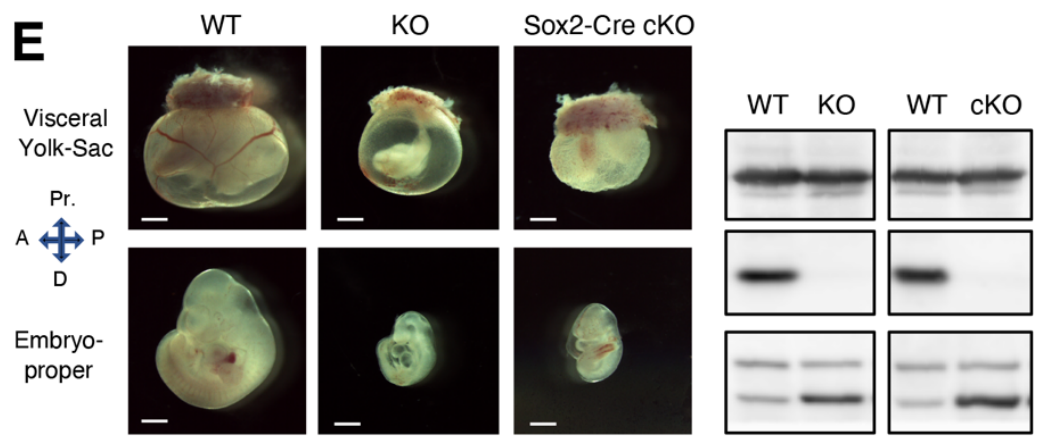

a-tubulin

Late Gastrulation (E10.5)

Figure 1: Embryo morphology and size evaluation of CNOT9 KO embryos alongside WT controls during (A) Bud and Headfold stages (E7.25-E7.75) (B) Early somite stages (E7.75 - E8.0) (C) Intermediate to Late Somite stages (E8.0 - E8.5) (D) and (E) Late gastrulation stages (E9.0 - E10.5). Western Blotting for PARP alongside corresponding stages indicates the extent of tissue death in KO embryos compared to WT control. Sox2-Cre cKO embryos indicate marginal delay in emergence of $\mathrm{KO}$ embryo phenotype. Scale: 500um 

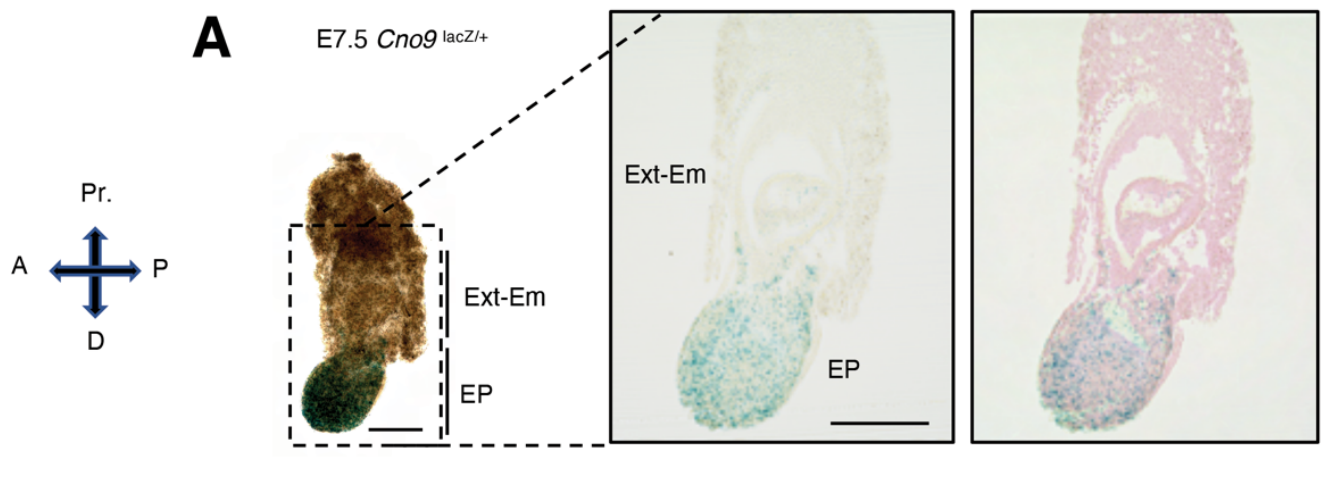

B


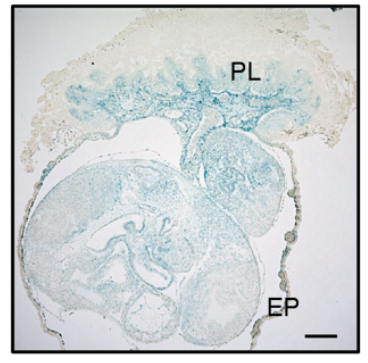

Axial section

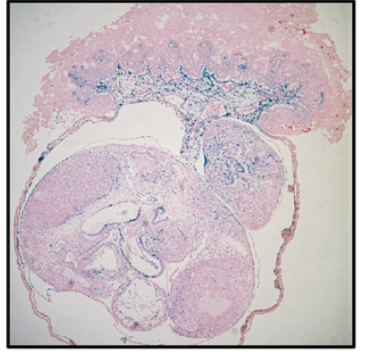

Axial section (eosin stained)

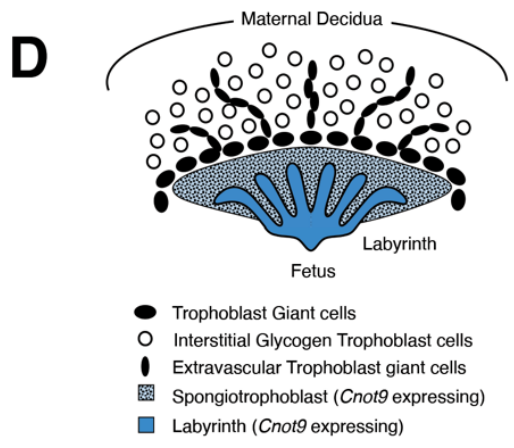

E

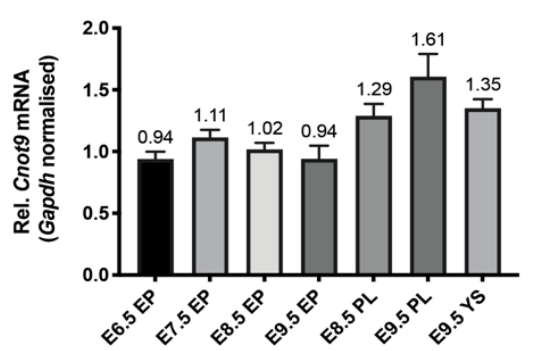

Figure 2: LacZ staining of CNOT9 HE embryos as a readout for spatial localization of Cnot9 mRNA. (A) E7.5 stage indicates majority of expression in epiblast derived regions of the embryo. (B) E8.5 stage depicts expression in various regions of the embryo-proper with marginal expression in regions of the ectoplacental cone. (C) E9.5 staged embryos show extensive expression in regions of the placenta that correspond to labyrinth and spongiotrophoblast cells. Embryo-proper also shows expression although not uniform across the embryo. (D) Schematic representation depicting Cnot9 expressing (LacZ stained) regions of the placenta in blue (E) Cnot9 mRNA levels, relative to Gapdh, detected by qRT-PCR in WT embryos in various embryo regions across stages of gastrulation. Schematic representation Scale: 250um, Ext-Em: extra embryonic, EPC: Ectoplacental cone, EP: Embryo-proper, PL: Placenta, YS: Yolk-sac, A: Anterior end, P: Posterior end, Pr.: Proximal end, D: 
A E8.5 KO vs WT gene expression (Tot. genes: 15534, Norm. RPKM >0.05)

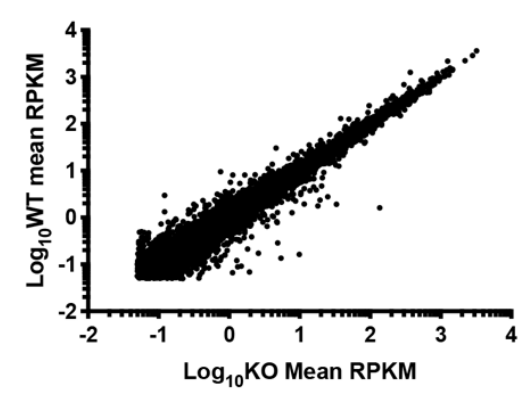

$>2$ fold change genes in E8.5 CNOT9 KO Embryo-proper

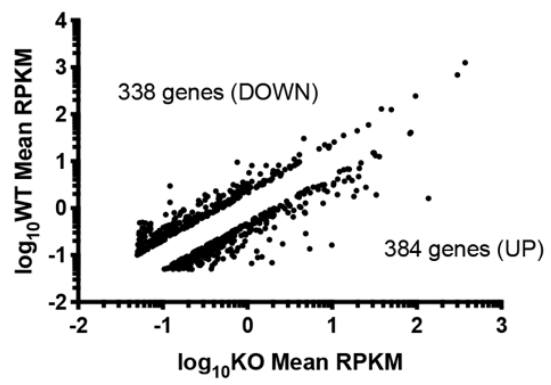

B Upregulated targets based on RNA-Seq data (RPKM normalized)

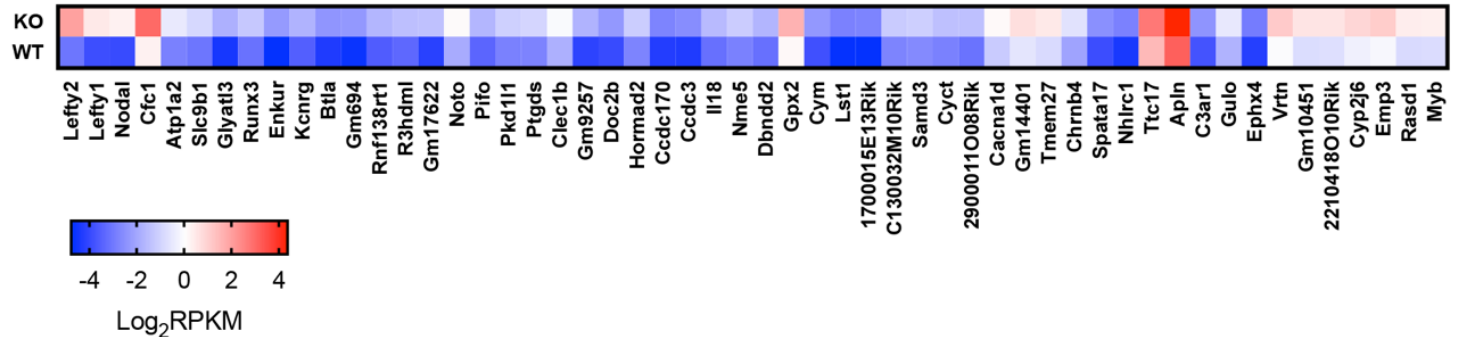

C Upregulated targets validated by qRT-PCR data (GAPDH normalized)
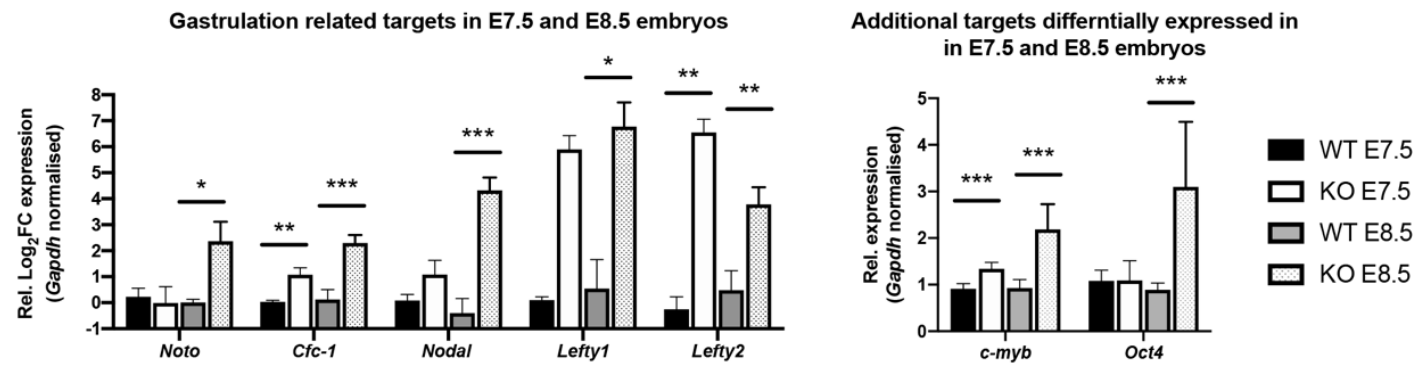

D Upregulated pathway (using Gene Set Enrichment Analysis):

\begin{tabular}{lccrrr}
\hline Gene Set Name/Description & $\begin{array}{c}\text { \# Genes in } \\
\text { Gene Set (K) }\end{array}$ & $\begin{array}{c}\text { \# Genes in } \\
\text { Overlap (k) }\end{array}$ & k/K & p-value & FDR q-value \\
Regulation of signaling by NODAL & 11 & 3 & 0.2727 & $7.41 \mathrm{E}-07$ & $2.50 \mathrm{E}-03$ \\
Signaling by NODAL & 20 & 3 & 0.15 & $5.07 \mathrm{E}-06$ & $8.56 \mathrm{E}-03$ \\
TGF-beta signaling pathway & 85 & 4 & 0.0471 & $1.33 \mathrm{E}-05$ & $1.50 \mathrm{E}-02$ \\
Genes defining inflammatory response & 200 & 5 & 0.025 & $2.26 \mathrm{E}-05$ & $1.91 \mathrm{E}-02$ \\
\hline
\end{tabular}

Figure 3: (A) Dot-plots representing gene expression in WT and KO embryos (left). More than 2-fold upregulated and downregulated targets (right) (B) Heat-map indicating average extent of upregulation for target genes in KO embryos compared to WT controls. (C) qRT-PCR based validation of upregulated genes involved in embryonic gastrulation in E7.5 and E8.5 stages $\left(n=5, p<0.05\left(^{*}\right), p<\right.$ $\left.\left.0.01{ }^{* \star}\right), p<0,001{ }^{(* \star}\right)$. (D) Key upregulated pathways in CNOT9 KO embryos mainly involve TGFbeta signaling pathways influenced by Nodal and other competing ligands 
A

Lefty2 3'UTR

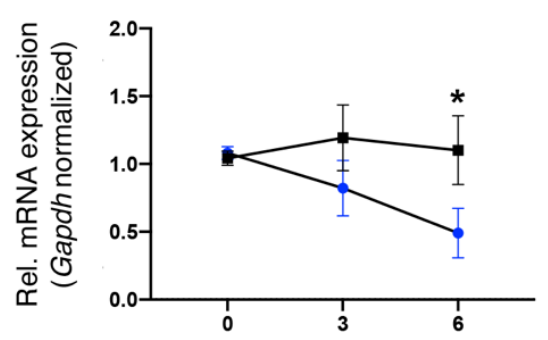

Lefty13'UTR

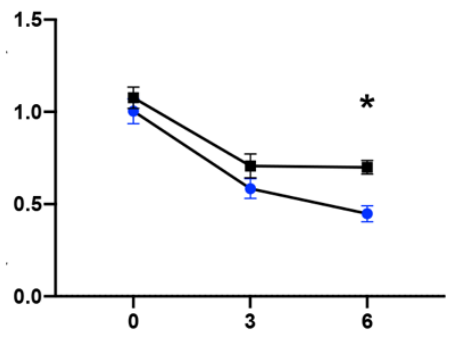

c-myb3'UTR

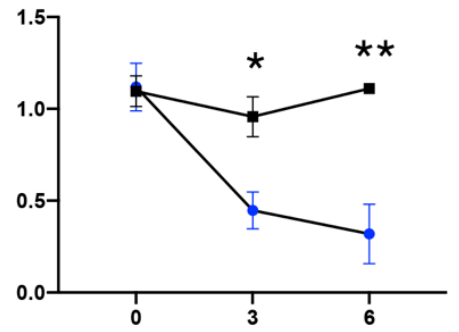

Hours of ActinomycinD treatment

B

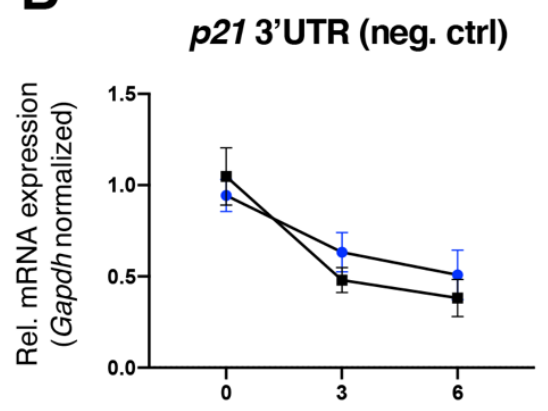

FHL3 (endogenous ctrl.)

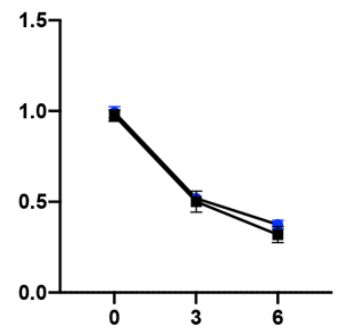

Hours of ActinomycinD treatment

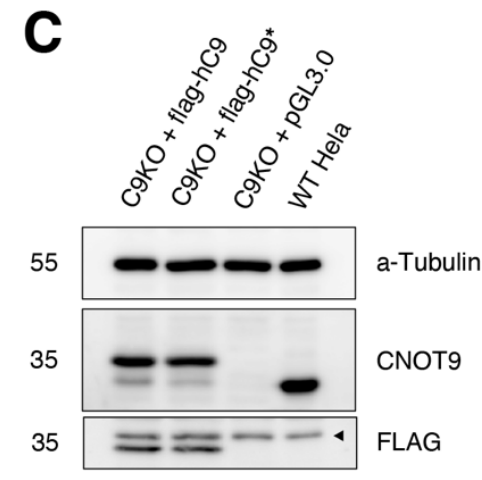

4 Non-specific band

- - CNOT9 KO HeLa + Flag-CNOT9 + Luc-3'UTR

$\rightarrow$ CNOT9 KO HeLa + Flag-CNOT9mut4 + Luc-3'UTR

Figure 4: Decay kinetics of Luciferase reporter conjugated with 3'UTR elements of (A) Lefty1, Lefty2, and c-myb (B) p21 3'UTR as non-target control and Fh/3 as endogenous control. Blue dots represent relative expression of reporter mRNA in CNOT9 KO cells reconstituted with human CNOT9, whereas black dots represent expression in CNOT9 KO cells reconstituted with mutant CNOT9 (CNOT9mut4) that cannot interact with CNOT1. (C) Western Blotting using anti-CNOT9 and anti-FLAG antibodies to ensure equal expression of CNOT9 and CNOT9mut4 proteins. Values are Mean $\pm S D\left[n=3, p<0.05\left(^{*}\right)\right.$, $\left.\mathrm{p}<0.01{ }^{(*)}\right]$ 


\section{References}

BASSON, M. A. 2012. Signaling in cell differentiation and morphogenesis. Cold Spring Harb Perspect Biol, 4.

CHAPAT, C. \& CORBO, L. 2014. Novel roles of the CCR4-NOT complex. Wiley Interdiscip Rev RNA, 5, 883-901.

CHEN, Y., BOLAND, A., KUZUOGLU-OZTURK, D., BAWANKAR, P., LOH, B., CHANG, C. T., WEICHENRIEDER, O. \& IZAURRALDE, E. 2014. A DDX6-CNOT1 complex and Wbinding pockets in CNOT9 reveal direct links between miRNA target recognition and silencing. Mol Cell, 54, 737-50.

CHU, J., DING, J., JEAYS-WARD, K., PRICE, S. M., PLACZEK, M. \& SHEN, M. M. 2005. Non-cell-autonomous role for Cripto in axial midline formation during vertebrate embryogenesis. Development, 132, 5539-51.

COLLART, M. A. 2016. The Ccr4-Not complex is a key regulator of eukaryotic gene expression. Wiley Interdiscip Rev RNA, 7, 438-54.

CONLON, F. L., LYONS, K. M., TAKAESU, N., BARTH, K. S., KISPERT, A., HERRMANN, B. \& ROBERTSON, E. J. 1994. A primary requirement for nodal in the formation and maintenance of the primitive streak in the mouse. Development, 120, 1919-28.

GARAPATY, S., MAHAJAN, M. A. \& SAMUELS, H. H. 2008. Components of the CCR4-NOT complex function as nuclear hormone receptor coactivators via association with the NRCinteracting Factor NIF-1. J Biol Chem, 283, 6806-16.

GILBERT, S. F. 2010. Developmental biology, Sunderland, Sinauer.

HAAS, M., SIEGERT, M., SCHURMANN, A., SODEIK, B. \& WOLFES, H. 2004. c-Myb protein interacts with Rcd-1, a component of the CCR4 transcription mediator complex. Biochemistry, 43, 8152-9.

HAMMERSCHMIDT, M. \& WEDLICH, D. 2008. Regulated adhesion as a driving force of gastrulation movements. Development, 135, 3625-41.

HIROI, N., ITO, T., YAMAMOTO, H., OCHIYA, T., JINNO, S. \& OKAYAMA, H. 2002. Mammalian Rcd1 is a novel transcriptional cofactor that mediates retinoic acid-induced cell differentiation. EMBO J, 21, 5235-44.

LABUN, K., MONTAGUE, T. G., KRAUSE, M., TORRES CLEUREN, Y. N., TJELDNES, H. \& VALEN, E. 2019. CHOPCHOP v3: expanding the CRISPR web toolbox beyond genome editing. Nucleic Acids Res, 47, W171-W174.

LIN, X., ZHAO, W., JIA, J., LIN, T., XIAO, G., WANG, S., LIN, X., LIU, Y., CHEN, L., QIN, Y., LI, J., ZHANG, T., HAO, W., CHEN, B., XIE, R., CHENG, Y., XU, K., YAO, K., HUANG, W., XIAO, D. \& SUN, Y. 2016. Ectopic expression of Cripto-1 in transgenic mouse embryos causes hemorrhages, fatal cardiac defects and embryonic lethality. Sci Rep, 6, 34501.

MA, H., LIN, Y., ZHAO, Z. A., LU, X., YU, Y., ZHANG, X., WANG, Q. \& LI, L. 2016. MicroRNA-127 Promotes Mesendoderm Differentiation of Mouse Embryonic Stem Cells by Targeting Left-Right Determination Factor 2. J Biol Chem, 291, 12126-35. 
MANDELBAUM, J., SHESTOPALOV, I. A., HENDERSON, R. E., CHAU, N. G., KNOECHEL, B., WICK, M. J. \& ZON, L. I. 2018. Zebrafish blastomere screen identifies retinoic acid suppression of MYB in adenoid cystic carcinoma. J Exp Med, 215, 2673-2685.

MATHYS, H., BASQUIN, J., OZGUR, S., CZARNOCKI-CIECIURA, M., BONNEAU, F., AARTSE, A., DZIEMBOWSKI, A., NOWOTNY, M., CONTI, E. \& FILIPOWICZ, W. 2014. Structural and biochemical insights to the role of the CCR4-NOT complex and DDX6 ATPase in microRNA repression. Mol Cell, 54, 751-65.

MENO, C., GRITSMAN, K., OHISHI, S., OHFUJI, Y., HECKSCHER, E., MOCHIDA, K., SHIMONO, A., KONDOH, H., TALBOT, W. S., ROBERTSON, E. J., SCHIER, A. F. \& HAMADA, H. 1999. Mouse Lefty2 and zebrafish antivin are feedback inhibitors of nodal signaling during vertebrate gastrulation. Mol Cell, 4, 287-98.

MENO, C., SHIMONO, A., SAIJOH, Y., YASHIRO, K., MOCHIDA, K., OHISHI, S., NOJI, S., $\mathrm{KONDOH}, \mathrm{H}$. \& HAMADA, H. 1998. lefty-1 is required for left-right determination as a regulator of lefty-2 and nodal. Cell, 94, 287-97.

MILLER, J. E. \& REESE, J. C. 2012. Ccr4-Not complex: the control freak of eukaryotic cells. Crit Rev Biochem Mol Biol, 47, 315-33.

MORITA, M., OIKE, Y., NAGASHIMA, T., KADOMATSU, T., TABATA, M., SUZUKI, T., NAKAMURA, T., YOSHIDA, N., OKADA, M. \& YAMAMOTO, T. 2011. Obesity resistance and increased hepatic expression of catabolism-related mRNAs in Cnot3+/- mice. EMBO J, 30, 4678-91.

NEELY, G. G., KUBA, K., CAMMARATO, A., ISOBE, K., AMANN, S., ZHANG, L., MURATA, M., ELMEN, L., GUPTA, V., ARORA, S., SARANGI, R., DAN, D., FUJISAWA, S., USAMI, T., XIA, C. P., KEENE, A. C., ALAYARI, N. N., YAMAKAWA, H., ELLING, U., BERGER, C., NOVATCHKOVA, M., KOGLGRUBER, R., FUKUDA, K., NISHINA, H., ISOBE, M., POSPISILIK, J. A., IMAI, Y., PFEUFER, A., HICKS, A. A., PRAMSTALLER, P. P., SUBRAMANIAM, S., KIMURA, A., OCORR, K., BODMER, R. \& PENNINGER, J. M. 2010. A global in vivo Drosophila RNAi screen identifies NOT3 as a conserved regulator of heart function. Cell, 141, 142-53.

PAVANELLO, L., HALL, B., AIRHIHEN, B. \& WINKLER, G. S. 2018. The central region of CNOT1 and CNOT9 stimulates deadenylation by the Ccr4-Not nuclease module. Biochem $\mathrm{J}$, $475,3437-3450$.

PEREZ-GARCIA, V., FINEBERG, E., WILSON, R., MURRAY, A., MAZZEO, C. I., TUDOR, C., SIENERTH, A., WHITE, J. K., TUCK, E., RYDER, E. J., GLEESON, D., SIRAGHER, E., WARDLE-JONES, H., STAUDT, N., WALI, N., COLLINS, J., GEYER, S., BUSCHNENTWICH, E. M., GALLI, A., SMITH, J. C., ROBERTSON, E., ADAMS, D. J., WENINGER, W. J., MOHUN, T. \& HEMBERGER, M. 2018. Placentation defects are highly prevalent in embryonic lethal mouse mutants. Nature, 555, 463-468.

PFENDLER, K. C., CATUAR, C. S., MENESES, J. J. \& PEDERSEN, R. A. 2005. Overexpression of Nodal promotes differentiation of mouse embryonic stem cells into mesoderm and endoderm at the expense of neuroectoderm formation. Stem Cells Dev, 14, 162-72. 
RAISCH, T., CHANG, C. T., LEVDANSKY, Y., MUTHUKUMAR, S., RAUNSER, S. \& VALKOV, E. 2019. Reconstitution of recombinant human CCR4-NOT reveals molecular insights into regulated deadenylation. Nat Commun, 10, 3173.

RAN, F. A., HSU, P. D., WRIGHT, J., AGARWALA, V., SCOTT, D. A. \& ZHANG, F. 2013. Genome engineering using the CRISPR-Cas9 system. Nat Protoc, 8, 2281-2308.

REIK, W. 2007. Stability and flexibility of epigenetic gene regulation in mammalian development. Nature, 447, 425-32.

SHIRAI, Y. T., SUZUKI, T., MORITA, M., TAKAHASHI, A. \& YAMAMOTO, T. 2014. Multifunctional roles of the mammalian CCR4-NOT complex in physiological phenomena. Front Genet, 5, 286.

TAKAHASHI, A., ADACHI, S., MORITA, M., TOKUMASU, M., NATSUME, T., SUZUKI, T. \& YAMAMOTO, T. 2015. Post-transcriptional Stabilization of Ucp1 mRNA Protects Mice from Diet-Induced Obesity. Cell Rep, 13, 2756-67.

TODOKORO, K., WATSON, R. J., HIGO, H., AMANUMA, H., KURAMOCHI, S., YANAGISAWA, H. \& IKAWA, Y. 1988. Down-regulation of c-myb gene expression is a prerequisite for erythropoietin-induced erythroid differentiation. Proc Natl Acad Sci U S A, $85,8900-4$.

TOMANCAK, P., BERMAN, B. P., BEATON, A., WEISZMANN, R., KWAN, E., HARTENSTEIN, V., CELNIKER, S. E. \& RUBIN, G. M. 2007. Global analysis of patterns of gene expression during Drosophila embryogenesis. Genome Biol, 8, R145.

WANG, J., SINHA, T. \& WYNSHAW-BORIS, A. 2012. Wnt signaling in mammalian development: lessons from mouse genetics. Cold Spring Harb Perspect Biol, 4.

WANG, S., COWAN, C. A., CHIPPERFIELD, H. \& POWERS, R. D. 2005. Gene expression in the preimplantation embryo: in-vitro developmental changes. Reprod Biomed Online, 10, 607-16.

YAGI, T., TOKUNAGA, T., FURUTA, Y., NADA, S., YOSHIDA, M., TSUKADA, T., SAGA, Y., TAKEDA, N., IKAWA, Y. \& AIZAWA, S. 1993. A novel ES cell line, TT2, with high germlinedifferentiating potency. Analytical biochemistry, 214, 70-6.

YAN, Y. T., GRITSMAN, K., DING, J., BURDINE, R. D., CORRALES, J. D., PRICE, S. M., TALBOT, W. S., SCHIER, A. F. \& SHEN, M. M. 1999. Conserved requirement for EGF-CFC genes in vertebrate left-right axis formation. Genes Dev, 13, 2527-37.

YI, H., XUE, L., GUO, M. X., MA, J., ZENG, Y., WANG, W., CAI, J. Y., HU, H. M., SHU, H. B., SHI, Y. B. \& LI, W. X. 2010. Gene expression atlas for human embryogenesis. FASEB J, $24,3341-50$. 